\title{
INTRINSIC AND REPROCESSED OPTICAL EMISSION FROM THE COMPANION TO PSR J2051-0827
}

\author{
B. W. Stappers, ${ }^{1}$ M. H. van Kerkwijk, ${ }^{2}$ J. F. Bell, ${ }^{3}$ and S. R. Kulkarni ${ }^{4}$ \\ Received 2000 October 13; accepted 2000 December 22; published 2001 February 19
}

\begin{abstract}
Hubble Space Telescope observations of the companion to the eclipsing millisecond pulsar PSR J2051-0827 have revealed its "dark" side. The $R$ magnitude at minimum is $\sim 26$, while the difference between the side heated by the impinging pulsar radiation and the unirradiated side is $\sim 3.3$ mag. For the first time, these data show that there is an asymmetry in the optical light curve of the companion. Furthermore, significant variability is seen in the companion brightness measured after optical maximum on successive orbits. The data are modeled by a gravitationally distorted low-mass secondary star that is irradiated by the pulsar wind. These model fits indicate that the system is only mildly inclined $\left(i \sim 40^{\circ}\right)$ and the unilluminated side of the companion has a temperature likely less than $3000 \mathrm{~K}$.
\end{abstract}

Subject headings: binaries: eclipsing — pulsars: individual (PSR J2051-0827) — stars: neutron

\section{INTRODUCTION}

The rapidly rotating subclass of pulsars known as millisecond pulsars are thought to have been regenerated through the process of spin-up by mass accretion from their companions during an X-ray binary phase (Alpar et al. 1982; Radhakrishnan $\&$ Srinivasan 1982). If isolated millisecond pulsars also form in this manner, then the companion needs to be removed; this might occur via a combination of Roche lobe overflow and ablation. Both these processes can occur at least partially during the X-ray phase (Ruderman et al. 1989) and then continue once accretion has stopped and the pulsar begins emitting in the radio (Rasio, Shapiro, \& Teukolsky 1989). The discovery of the first eclipsing binary millisecond pulsar B1957+20, where there is clear evidence for the ablation of the companion star (Fruchter, Stinebring, \& Taylor 1988), and the more recent discovery of SAX 1808.4-3658, an X-ray pulsar spinning at millisecond periods (Wijnands \& van der Klis 1998; Chakrabarty \& Morgan 1998), seem to confirm this formation scenario.

PSR J2051-0827 is the second known eclipsing binary millisecond pulsar with an optically identified companion (Stappers, Bessell, \& Bailes 1996a). It has a spin period of $4.5 \mathrm{~ms}$ and an orbital period of 0.1 days (Stappers et al. 1996b). There is evidence that the eclipse region extends beyond the Roche lobe of the companion. Radio timing studies yield a lower limit on the companion mass of $0.025 M_{\odot}$, but no information on its radius relative to its Roche lobe. Optical observations of the companion provide the best hope of determining this radius. Furthermore, modeling of the light curve can provide information on the degree of heating of the companion star and thus on how much energy is available for driving a wind from the companion. It is also possible to place strong constraints on the inclination of the system and therefore on the companion mass and the true extent of the eclipse region. A determination of the companion mass and radius will constrain the nature of the companion, such as whether it is degenerate or not.

The light curve of PSR J2051-0827 has been studied pre-

\footnotetext{
${ }^{1}$ Sterrenkundig Instituut "Anton Pannekoek," Universiteit van Amsterdam, Kruislan 403, 1098 SJ Amsterdam, The Netherlands; bws@ astro.uva.nl.

${ }^{2}$ Astronomical Institute, Utrecht University, P.O. Box 80000, 3508 TA Utrecht, The Netherlands; m.h.vankerkwijk@astro.uu.nl.

${ }^{3}$ Australia Telescope National Facility, CSIRO, P.O. Box 76, Epping 1710, NSW, Australia; jbell@atnf.csiro.au.

${ }^{4}$ Palomar Observatory, MS 105-24, California Institute of Technology, Pasadena, CA 91125
}

viously (Stappers et al. 1996a, 1999), and the companion was modeled by a gravitationally distorted star, irradiated by the pulsar wind. These results indicate that $30 \%$ of the pulsar's spin-down energy, assumed to be in the form of an isotropic relativistic pulsar wind, which is incident on the companion star, is reradiated as optical emission. Furthermore, the companion star may underfill its Roche lobe. This provides evidence that (at present in this system) the pulsar wind is the cause of evaporation of the companion star rather than tidal dissipation through Roche lobe overflow. A limitation of this earlier study is that it was only possible to observe the portion of the companion irradiated by the pulsar. The best constraints on the stellar radius and therefore on the geometry of the system and ablation models would come from modeling the complete light curve, including the cold unirradiated side.

In this Letter, we present Hubble Space Telescope (HST) observations in which we detect the unirradiated side of the companion and model the complete light curve. We also show that there is evidence for variability in the light curve and the presence of an extra source of emission.

\section{OBSERVATIONS AND MODELING}

The HST Wide Field Planetary Camera 2 (WFPC2) was used to obtain a total of 35 images of the PSR J2051-0827 field, spread over 12 orbits of the system on 1997 June 24, 25, 28, 29, and 30 and 1997 July 4 and 10. Details of filters and orbital phases are given in Table 1. Observation times were chosen to maximize orbital phase coverage when the companion is faintest. Exposure times were either 400 or $500 \mathrm{~s}$ and were chosen so as to enhance the chance of detection but limit the variation between each observation. The pointings were arranged so that the pulsar companion is placed on a portion of the Planetary Camera (PC) that was free from dead or hot pixels. Only the PC images are used in our analysis. Analysis of the images was completed using the IRAF package starting from the pipeline-calibrated PC images. One of the 35 images was rejected for further analysis due to a cosmic-ray strike precisely at the position of the companion. Cosmic-ray masks were generated by median combining observations from each orbit. The removal of cosmic rays is most important in this case for determining accurately the sky contribution in the region of the companion.

Aperture photometry was performed following the prescription of Holtzman et al. (1995). The faintness of the companion 
TABLE 1

SUMMARY OF HST OBSERVATIONS OF PSR J2051-0827

\begin{tabular}{|c|c|c|}
\hline Phase & Magnitude & Error \\
\hline \multicolumn{3}{|c|}{ F675W } \\
\hline $0.012 \ldots \ldots$ & 25.6 & 0.4 \\
\hline $0.049 \ldots \ldots$ & 25.8 & 0.5 \\
\hline $0.068 \ldots \ldots$ & 25.1 & 0.3 \\
\hline $0.106 \ldots \ldots$ & 25.0 & 0.3 \\
\hline $0.107 \ldots \ldots$ & 25.3 & 0.3 \\
\hline $0.145 \ldots \ldots$ & 24.5 & 0.2 \\
\hline $0.163 \ldots \ldots$ & 24.0 & 0.1 \\
\hline $0.163 \ldots \ldots$ & 23.9 & 0.1 \\
\hline $0.201 \ldots \ldots$ & 23.8 & 0.1 \\
\hline $0.263 \ldots \ldots$ & 23.2 & 0.1 \\
\hline $0.736 \ldots \ldots$ & 22.62 & 0.05 \\
\hline $0.792 \ldots \ldots$ & 23.17 & 0.07 \\
\hline $0.849 \ldots \ldots$ & 24.4 & 0.2 \\
\hline $0.849 \ldots \ldots$ & 23.8 & 0.1 \\
\hline $0.853 \ldots \ldots$ & 23.9 & 0.1 \\
\hline $0.905 \ldots \ldots$ & 24.7 & 0.2 \\
\hline $0.967 \ldots \ldots$ & 25.6 & 0.3 \\
\hline $0.967 \ldots \ldots$ & 24.6 & 0.2 \\
\hline $0.993 \ldots \ldots$ & 25.2 & 0.3 \\
\hline \multicolumn{3}{|c|}{ F814W } \\
\hline $0.006 \ldots \ldots$ & 24.0 & 0.1 \\
\hline $0.015 \ldots \ldots$ & 24.1 & 0.2 \\
\hline $0.062 \ldots \ldots$ & 24.0 & 0.1 \\
\hline $0.077 \ldots \ldots$ & 23.9 & 0.1 \\
\hline $0.124 \ldots \ldots$ & 23.7 & 0.1 \\
\hline $0.224 \ldots \ldots$ & 22.85 & 0.07 \\
\hline $0.280 \ldots \ldots$ & 22.54 & 0.06 \\
\hline $0.745 \ldots \ldots$ & 22.23 & 0.04 \\
\hline $0.801 \ldots \ldots$ & 22.53 & 0.06 \\
\hline $0.873 \ldots \ldots$ & 23.4 & 0.1 \\
\hline $0.878 \ldots \ldots$ & 23.7 & 0.1 \\
\hline $0.929 \ldots \ldots$ & 23.8 & 0.1 \\
\hline $0.934 \ldots \ldots$ & 23.8 & 0.1 \\
\hline $0.959 \ldots \ldots$ & 24.0 & 0.1 \\
\hline $0.996 \ldots \ldots$ & 24.1 & 0.1 \\
\hline
\end{tabular}

Note.-Binary phase is defined such that phase zero occurs when the companion lies between the Earth and the pulsar. meant that the best signal-to-noise ratio is obtained for relatively smaller apertures. Apertures of different radii were used on a number of brighter stars in the field to determine aperture corrections relative to the standard 0"5 (11 pixel) radius aperture. In Table 1, we present these aperture-corrected magnitudes, which have been transferred to the Vega system by applying a $0.10 \mathrm{mag}$ aperture correction from 0.5 radius to "nominal infinity" (Baggett et al. 1997). The corrections for losses due to the charge-transfer efficiency were made following the prescription of Whitmore, Heyer, \& Casertano (1999). No correction has been made for the so-called long versus short anomaly as there is presently no consensus on the nature of this problem. A recent study by Dolphin (2000) suggests that the problem is caused by an incorrect determination of the background in short exposures. Our exposures have a welldetermined background, and so we believe our magnitudes are unaffected by this problem. However, we note that if a correction to our magnitudes of the size suggested ${ }^{5}$ is required, then our data would need to be remodeled.

As a check on our measurements and transformations, F675W magnitudes were determined for a faint comparison star (of similar flux to the mean flux of the PSR J2051-0827 companion) detected on the PC. Assuming that this star was intrinsically constant in flux, we obtained a reduced $\chi^{2}=$ 1.06, indicating that the uncertainties obtained for the magnitudes are realistic. Previous observations from the Hale 200 inch and the Anglo-Australian Telescope that are also used in the following analysis have been described in detail by Stappers et al (1999). The complete data set of 53 observations (shown in Fig. 1) was phased using the known ephemeris for the binary orbit and then modeled. We note here that there is excellent agreement between the ground-based magnitudes and the HST magnitudes converted to Cousins $R$ and $I$.

A model of a synchronously rotating companion star irradiated by the impinging pulsar wind that includes the effects of gravitational and rotational distortion and gravity darkening

${ }^{5}$ See the 1999 June update to the WFPC2 Instrument Handbook for Cycle 9.
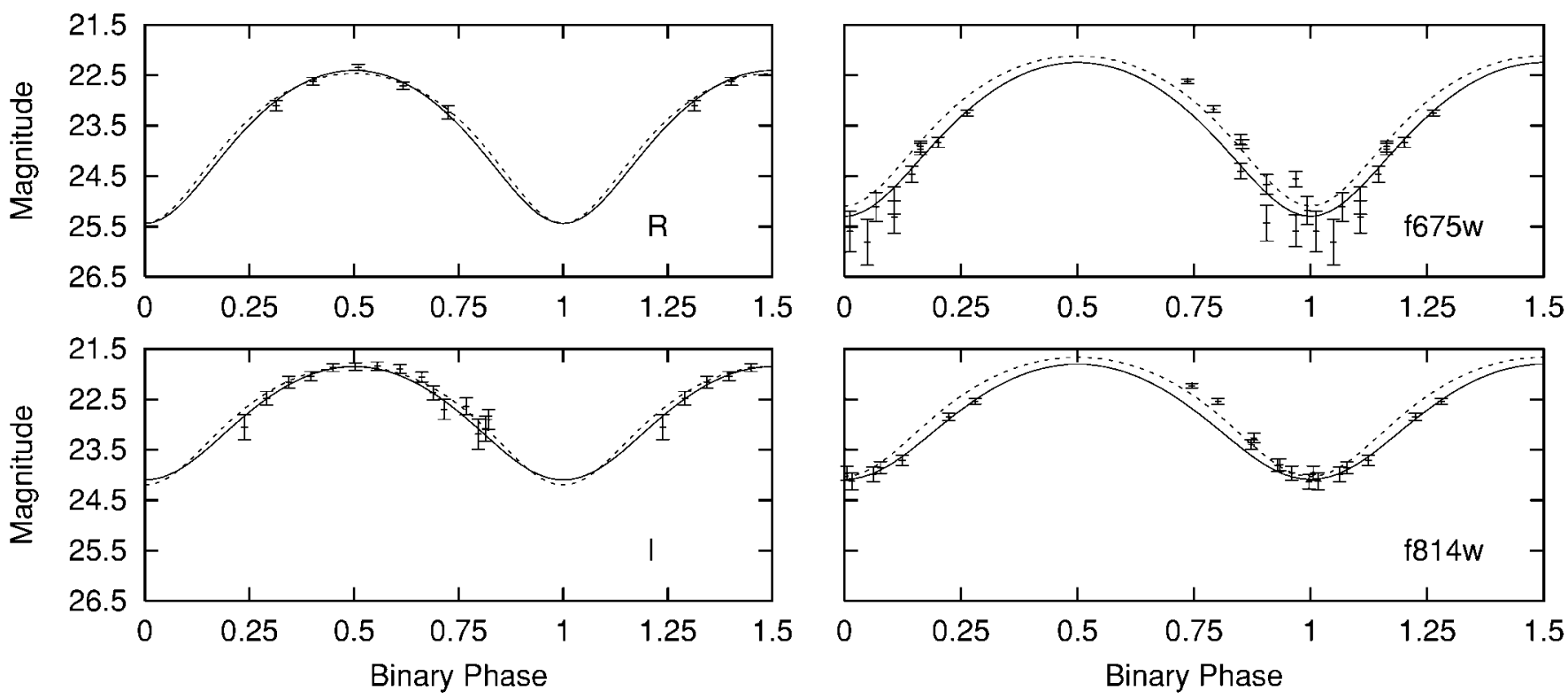

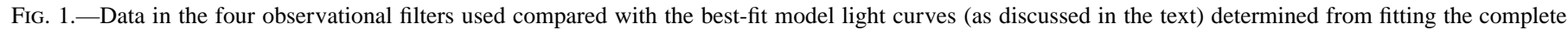
light curve (dashed lines) and fitting only the rising part of the light curve (solid lines). 


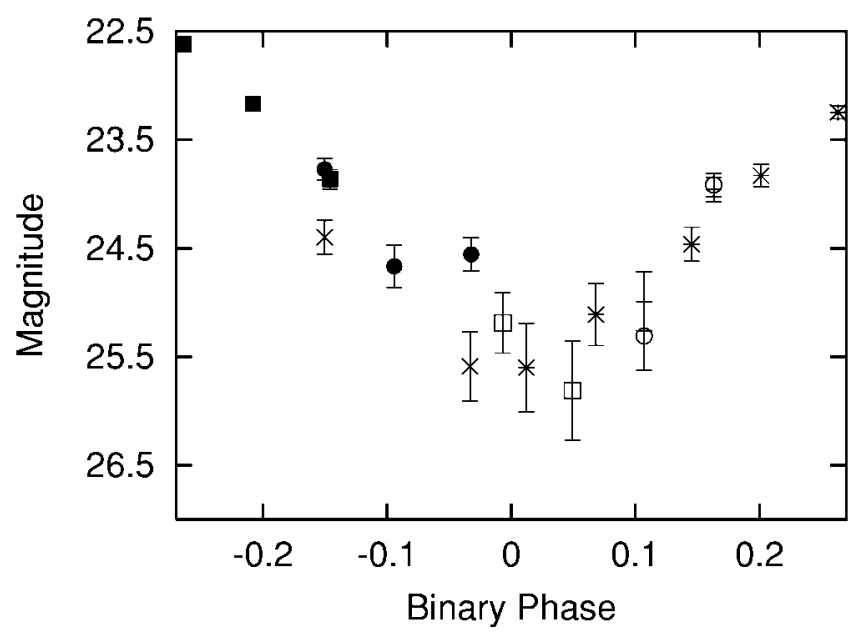

FIG. 2.-All observations made in the F675W HST filter. Groups of magnitudes that were measured during the same system orbit are indicated by the same symbol. In total, there are data from seven orbits shown.

and the inclination of the binary orbit is fitted to the data. The model also fits for the distance modulus and the extinction in the direction of the source. As was discussed in Stappers et al. (1999), we use the dispersion measure-determined distance and an extinction determined from IRAS maps, and their associated errors, to constrain these two parameters to $(m-M)_{0}=$ $10.6 \pm 0.6$ and $E_{B-V}=0.06 \pm 0.03$, respectively. As the unirradiated side of the companion star is likely very cool, the emergent spectrum needs to be modeled carefully. Thus, the model magnitudes were calculated using the most recent lowtemperature stellar atmosphere models of Hauschildt, Allard, \& Baron (1999) and folding them through the appropriate individual filter functions. Note that the Cousins $I$ and $R$ data and that recorded in the HST filters were fitted simultaneously (i.e., the offsets between the magnitudes were considered by folding through the appropriate filter functions).

\section{DISCUSSION}

A closer inspection of the phased light curve shown in Figure 1 shows two important features that were only hinted at by the ground-based observations. First, there is an asymmetry apparent in the light curve where the decrease in brightness to minimum occurs more steeply than does the increase to maximum brightness. In other words, the presence of excess emission after maximum means that the decrease to minimum is apparent at later phases than would be expected for a symmetric light curve. Second, the brightness of the companion varies significantly between orbital phases 0.5 and 1.0 in successive orbits. Such large variations in brightness are not observed in the other half of the orbit. This can be seen most clearly in Figure 2, where all seven orbits obtained in the F675W filter are shown. The amplitude of these variations is as large as $1 \mathrm{mag}$, as seen between the observations indicated by the filled circles and the crosses in Figure 2.

Modeling of the light curve is of course limited by these complications; a simple synchronously rotating companion resulting in a symmetric light curve is no longer a complete model. If we fit the complete data set using a symmetric model, we find that we overestimate the flux in the rising part of the light curve. The resultant best-fit model is of a Roche lobe-filling companion star which converts approximately $30 \%$
TABLE 2

Fit Parameters

\begin{tabular}{lll}
\hline \hline \multicolumn{1}{c}{ Parameter } & Phases $0.0-1.0$ & Phases $0.0-0.5$ \\
\hline Irradiation efficieny $\ldots \ldots$. & $0.3_{-0.07}^{+0.2}$ & $0.45_{-0.1}^{+0.2}$ \\
Filling factor $\ldots \ldots \ldots \ldots$. & $0.95_{-0.02}^{+0.05}$ & $0.43_{-0.16}^{+0.23}$ \\
Temperature $(\mathrm{K}) \ldots \ldots \ldots$. & $2500_{-150}^{+900}$ & $2800_{-200}^{+600}$ \\
Inclination $(\mathrm{deg}) \ldots \ldots \ldots$. & $38_{-2}^{+20}$ & $40_{-4}^{+9}$ \\
Reduced $\chi^{2} \ldots \ldots \ldots \ldots \ldots$ & 5.6 & 0.96 \\
\hline
\end{tabular}

of the incident pulsar spin-down energy into optical flux. It is important to note that the distance and extinction do play a strong role when determining the radius. The range of valid distances and extinction values allowed are dependent on the overall quality of the fit. As this quality is lower in this case than for the fit of Stappers et al. (1999), the radius is also less well constrained.

Alternatively, we can consider that the variability and asymmetry that is seen during the falling part of the light curve is associated with some other source of emission. Assuming the underlying system must be synchronously rotating and evenly heated (i.e., no hot spots) and thus has a symmetric light curve, we fit just the rising part of the light curve. This results in a model with a significantly improved fit (see Table 2). The companion star fills less than half its Roche lobe and converts up to $15 \%$ more of the incident spin-down energy into optical emission. The results of this fit are similar to the original model discussed by Stappers et al. (1999).

The important fit parameters are the following: irradition efficiency - the percentage of the pulsar's spin-down energy, assumed to be radiated isotropically, which is reradiated as optical emission from the companion; the filling factor-the ratio of the radii of spheres of equivalent volume to the distorted companion radius and the Roche lobe radius; the temperature of the unirradiated face of the companion star; and the inclination of the plane of the orbit to the sky. The values of these parameters and their $1 \sigma$ uncertainties are shown for both fits in Table 2. Despite the limitations on the fitting discussed above, three parameters are relatively strongly constrained. The initially most surprising constraint is that on the inclination. This system is only moderately inclined, that is, $i \sim 40^{\circ}$, which corresponds to a mass for the companion star of $\sim 0.04 \mathrm{M}_{\odot}$. A low inclination may help to explain the strong frequency dependence and large variability observed in the radio eclipses (Stappers et al. 2001), since in this case the radio beam intercepts only the outer limits of the wind. It is also apparent that a large fraction, greater than $30 \%$, of the pulsar's spin-down energy is converted to optical emission and the backside temperature of the star is very cool, likely less than $3000 \mathrm{~K}$.

Spectroscopic observations and photometry of a number of contiguous orbits is required to understand better the cause of the asymmetry and variability in the light curve. If some fraction of the emission that we see is coming from a shock above the companion's surface, then that would clearly show up in a spectrum of the system. The timescale for the variability can be determined only by more intensive observing sessions than those possible with the observing constraints of the HST.

The observations were obtained with the NASA/ESA Hubble Space Telescope at the Space Telescope Science Institute, which is operated by the Association of Universities for Research in Astronomy, Inc. The reduction of the optical data was done using the Image Reduction and Analysis Facility (IRAF), which is developed and maintained by the National 
Optical Astronomy Observatories. We acknowledge support of a NASA Guest Observer grant, a fellowship of the Royal Netherlands Academy of Arts and Sciences (M. H. v. K.), and an
Australian Research Council Fellowship (J. F. B.); B. W. S. is supported by NWO Spinoza grant 08-0 to E. P. J. van den Heuvel.

\section{REFERENCES}

Alpar, M. A., Cheng, A. F., Ruderman, M. A., \& Shaham, J. 1982, Nature, 300, 728

Baggett, S., Casertano, S., Gonzaga, S., \& Ritchie, C. 1997, WFPC2 Instrument Science Report 97-10 (Baltimore: STScI)

Chakrabarty, D., \& Morgan, E. H. 1998, Nature, 394, 346

Dolphin, A. E. 2000, PASP, 112, 1397

Fruchter, A. S., Stinebring, D. R., \& Taylor, J. H. 1988, Nature, 333, 237

Hauschildt, P. H., Allard, F., \& Baron, E. 1999, ApJ, 512, 377

Holtzman, J. A., et al. 1995, PASP, 107, 156

Radhakrishnan, V., \& Srinivasan, G. 1982, Curr. Sci., 51, 1096
Rasio, F. A., Shapiro, S. L., \& Teukolsky, S. A. 1989, ApJ, 342, 934

Ruderman, M., Shaham, J., Tavani, M., \& Eichler, D. 1989, ApJ, 343, 292

Stappers, B. W., Bailes, B., Lyne, A. G., Camilo, F., Manchester, R. N., Sandhu, J. S., Toscano, M., \& Bell, J. F. 2001, MNRAS, 321, 576

Stappers, B. W., Bessell, M. S., \& Bailes, M. 1996a, ApJ, 473, L119

Stappers, B. W., van Kerkwijk, M. H., Lane, B., \& Kulkarni, S. R. 1999, ApJ, 510, L45

Stappers, B. W., et al. 1996b, ApJ, 465, L119

Whitmore, B., Heyer, I., \& Casertano, S. 1999, PASP, 111, 1559

Wijnands, R., \& van der Klis, M. 1998, Nature, 394, 344 